\title{
Disproportionate Frequency Representation in the Inferior Colliculus of Doppler-Compensating Greater Horseshoe Bats : Evidence for an Acoustic Fovea*
}

\author{
Gerd Schuller ${ }^{1}$ and George Pollak ${ }^{2}$ \\ ${ }^{1}$ Arbeitskreis Neuro- und Rezeptorphysiologie, Fachbereich Biologie, Universität Frankfurt, D-6000 Frankfurt, \\ Federal Republic of Germany \\ ${ }^{2}$ Department of Zoology, The University of Texas, Austin, Texas 78712, USA
}

Accepted March 16, 1979

Summary. 1. The inferior colliculus of 8 Greater Horseshoe bats (Rhinolophus ferrumequinum) was systematically sampled with electrode penetrations covering the entire volume of the nucleus. The best frequencies and intensity thresholds for pure tones (Fig. 2) were determined for 591 neurons. The locations of the electrode penetrations within the inferior colliculus were histologically verified.

2. About $50 \%$ of all neurons encountered had best frequencies $(\mathrm{BF})$ in the frequency range between 78 and $88 \mathrm{kHz}$ (Table 1, Fig. 1A). Within this frequency range the BFs between 83.0 and $84.5 \mathrm{kHz}$ were overrepresented with $16.3 \%$ of the total population of neurons (Fig. 1B). The frequencies of the constant frequency components of the echoes fall into this frequency range.

3. The representation of BFs expressed as number of neurons per octave shows a striking correspondence to the nonuniform innervation density in the afferent innervation of the basilar membrane (Bruns and Schmieszek, in press). The high innervation density of the basilar membrane in the frequency band between 83 and $84.5 \mathrm{kHz}$ coincides with the maximum of the distribution of number of neurons per octave across frequency in the inferior colliculus (Fig. 1C).

4. The disproportionate representation of frequencies in the auditory system of the greater horseshoe bat is described as an acoustical fovea functioning in analogy to the fovea in the visual system. The functional importance of the Doppler-shift compensation for such a foveal mechanism in the auditory

* Supported by the Alexander von Humboldt Foundation, grant to G. Pollak; by Stiftung Volkswagenwerk, AZ.: 111858 and by the Deutsche Forschungsgemeinschaft, grants No. Schu 390/1,2 and $\operatorname{Br} 593 / 2$

Abbreviations: $B F$, best frequency; $C F$, constant frequency; $D S C$, Doppler-shift compensation; $F M$, frequency modulated system of horseshoe bats is related to that of tracking eye movements in the visual system.

\section{Introduction}

The long CF-FM bats belong to a taxonomically heterogeneous group characterized by three features: 1 ) distinctive echolocation cries; 2) a unique form of behaviour called Doppler-shift compensation (DSC); and 3) sharply tuned auditory systems. Each of these features is. well developed in the greater horseshoe bat, Rhinolophus ferrumequinum. Horseshoe bats emit orientation calls composed of a long constant frequency (CF) component followed by a brief downward sweeping FM portion (Schnitzler, 1967, 1968; Schuller et al., 1974). The CF-components emitted by these bats are typically $30-70 \mathrm{~ms}$ in duration and have frequencies between 82 and $85 \mathrm{kHz}$. However, it is important to note that each individual bat emits a highly stereotyped frequency which only varies $\pm 100 \mathrm{~Hz}$ from pulse to pulse. Under most conditions, echoes heard by the bat will be higher in frequency than the emitted cries due to the Doppler-shifts resulting from the approach. CF-FM bats exposed to Doppler-shifted echoes lower the frequency of their subsequent cries by an amount almost equal to the Doppler-shift (Schnitzler, 1968; Schuller et al., 1974; Simmons, 1974). As a consequence of DSC, echoes are held within a remarkably narrow band of frequencies only $\pm 50-100 \mathrm{~Hz}$ wide. The frequency at which the bat stabilizes its echoes is called the reference frequency and is typically $100-300 \mathrm{~Hz}$ higher than the resting frequency, i.e., the frequency emitted by the bat when it is not receiving Doppler-shifted echoes.

In conjunction with DSC is a population of exceptionally sharply tuned neurons (Suga et al., 1975, 
1976; Neuweiler and Vater, 1976; Möller et al., 1978). Whereas the $\mathrm{Q}_{10 \mathrm{~dB}}$-values are as high as $50-200$ and some as high as $400-500$, all of the sharply tuned neurons have best frequencies ranging from 78 to $88 \mathrm{kHz}$ and correspond to the lowered frequencies of the cries emitted during DSC and the full range of echoes the bat receives while hunting. Moreover, the frequencies between 78 and $88 \mathrm{kHz}$ are disproportionally represented along the entire auditory pathway, from the cochlea to the cortex (Bruns, 1976a, 1976b; Bruns and Schmieszek, in press; Möller et al., 1978; Schlegel, 1977; Suga et al., 1975, 1976; Suga and Jen, 1977).

To complete the picture, the thresholds of the filter neurons are not uniformly low but rather show a marked distribution that seemingly correlates well with DSC. Neurons tuned around the reference frequency are typically $20-40 \mathrm{~dB}$ lower in threshold than are other neurons having BFs both above and below the reference frequency (Möller et al., 1978; Schlegel, 1977; Suga et al., 1976).

Current ideas concerning the functional significance of the specialized features hold that the system is utilized primarily for evaluating relative velocity (Pollak et al., 1972; Schnitzler, 1973; Simmons et al., 1975; Suga et al., 1975, 1976; Suga, 1978a, b). The rationale is that due to the narrow bandwidth, constant frequency signals are poorly suited for target characterization but, for the same reason, they are well suited for Doppler measurements (Simmons et al., 1975; Simmons et al., 1979; Suga, 1978). Furthermore, since the long CF-FM bats Doppler-compensate, it is argued that they are analyzing relative velocity information. For processing this information, the sharply tuned neurons serve to spacially segregate the activity evoked by the emitted cry from the activity evoked by the Doppler-shifted echoes and permit fine frequency analysis of the pulse-echo frequency differences which is necessary for an evaluation of the relative velocity. The importance of DSC is that the bat holds the echo at a frequency to which the filter neurons exhibit the lowest thresholds, and in this way the bat is insured that echoes will be processed by a highly sensitive neuronal population (Grinnel1, 1973; Neuweiler, 1970; Pollak et al., 1972; Schnitzler, 1970, 1973; Suga et al., 1976). Many reports also add that the sharply tuned neurons encode the wing beat pattern of a flying insect by the synchronization of the discharges with the modulation pattern imposed on the echoes of the CF component (Schuller, 1972, 1979; Schnitzler, 1978; Suga and Jen, 1977). Since the statement is usually made that the CF signal is poorly suited for target characterization and since the question of prey recognition is only briefly mentioned, one concludes that many workers placed ma- jor emphasis on relative velocity measurements and consider the encoding of wing beat information to be of secondary importance.

During the course of monitoring single unit activities from the inferior colliculus (IC) of the Greater Horseshoe bat, Rhinolophus ferrumequinum, we obtained information concerning the distribution of BFs and thresholds from a large population of neurons. Some aspects of these data differ from previous reports and have an important bearing on questions concerned with the functional advantage of DSC as well as current ideas regarding the importance of evaluating relative velocity.

\section{Methods}

Details of the methods used have been reported elsewhere (Schuller, 1979). Briefly, 8 Rhinolophus ferrumequinum were prepared under Metofane anesthesia and were allowed to recover before experiments were begun. Single units were recorded with micropipettes filled with $3 \mathrm{M} \mathrm{KCl}$ and $3 \%$ Alcian blue dye (Harnischfeger, in press). The electrodes were advanced with an electronic, remote microdrive unit and the depth of each neuron was recorded. Actionpotentials were amplified with conventional techniques and sent to an oscilloscope and audiomonitor. Small spots of Alcian blue dye were deposited iontophoretically in each electrode tract, and at the end of each experiment the animals were sacrificed with an overdose of anesthetic. The brains were sectioned and each electrode tract was histologically verified as being confined to the inferior colliculus. From a systematic sampling of the inferior colliculus in the dorsoventral, anterior-posterior, and mediolateral axis 591 neurons responding to tone bursts were recorded. The BF of each neuron was determined audiovisually. No particular region was given preference either in terms of number of penetrations or number of units sampled.

\section{Results}

In Fig. $1 \mathrm{~A}$ is shown the distribution of BFs among the population of neurons encountered. The most striking feature of this histogram is the very large number of neurons tuned to frequencies between 78 and $88 \mathrm{kHz}$. Furthermore, it should be noted that neurons were responsive to frequencies covering at least three octaves from 9 to $96 \mathrm{kHz}$, but the neurons with BFs between 78 and $88 \mathrm{kHz}$ comprise $50 \%$ of the total population (Table 1). Consequently, about half of the neuronal population is devoted to only $12 \%$ of the entire audible frequency range (Long and Schnitzler, 1975), a finding in agreement with previous studies.

Of central importance is that the frequencies are not equally represented among the population of neurons between 78 and $88 \mathrm{kHz}$. Figure $1 \mathrm{~B}$ shows the distribution of cells where the BFs are plotted in intervals of $200 \mathrm{~Hz}$. Three features of this histogram are 

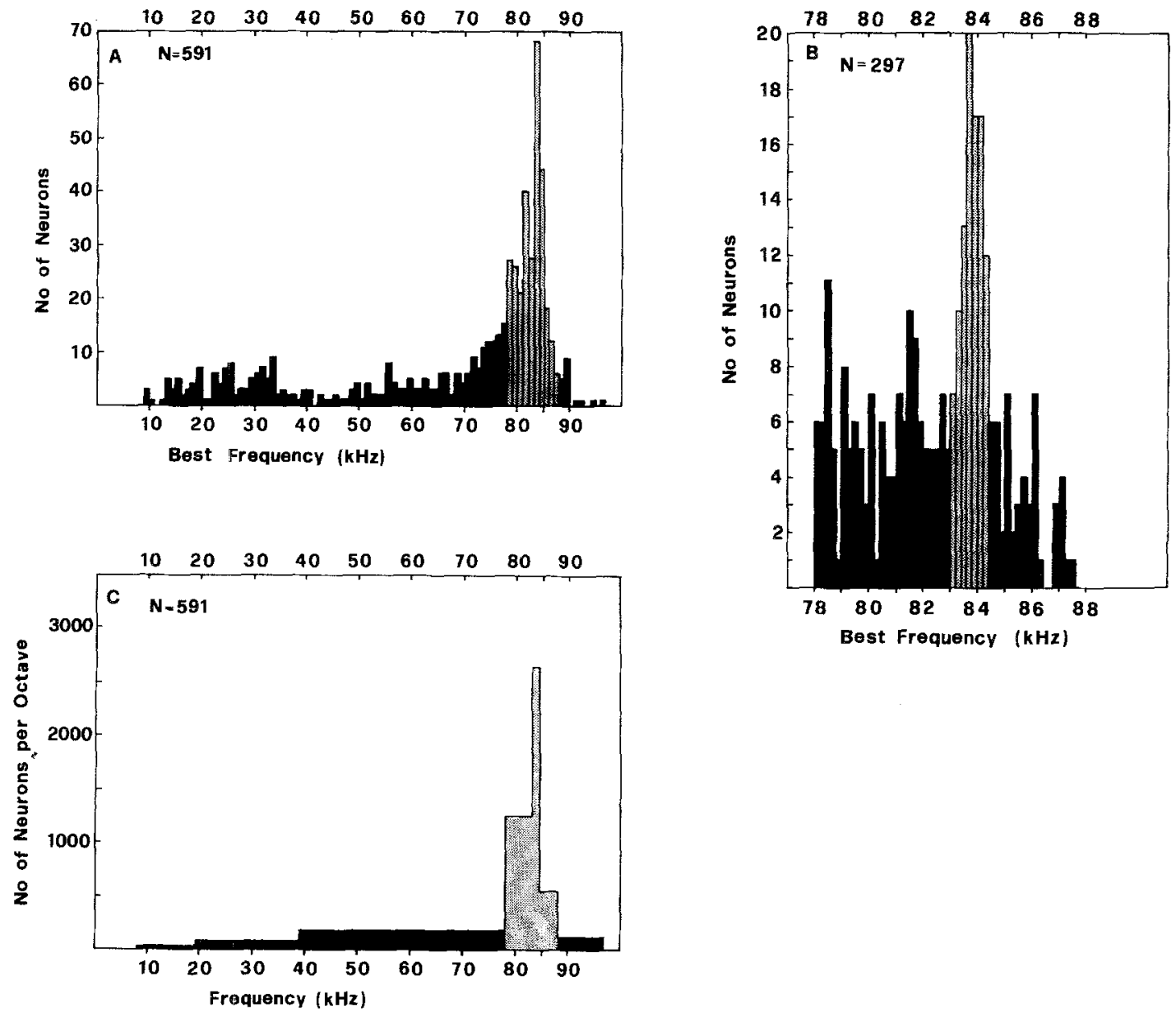

Fig. 1. A Histogram showing the number of neurons tuned to the frequencies comprising the audible range of greater horseshoe bats. Best frequencies are grouped and plotted in intervals of $1.0 \mathrm{kHz}$. The significant feature is the very large number of neurons tuned to the frequencies between $78-88 \mathrm{kHz}$. B Histogram showing the distribution of neurons tuned to the frequencies between 78 and $88 \mathrm{kHz}$ where the best frequencies are grouped and plotted in intervals of $200 \mathrm{~Hz}$. Note the fairly uniform number of neurons tuned to each of the frequency bands between $78.0-82.9 \mathrm{kHz}$ which is in marked contrast with the sharp increase in the number of neurons tuned between $83.0-84.5 \mathrm{kHz}$. An equally sharp decrease in the number of neurons tuned to frequencies above $84.5 \mathrm{kHz}$ is also evident. C Representation of best frequencies expressed as number of neurons per octave. Such a representation allows a direct comparison with innervation densities at the peripheral level of the basilar membrane (Bruns and Schmieszek, in press)

noteworthy: First, neurons having BFs ranging from 78 to $82.9 \mathrm{kHz}$ are about equally represented within this $4.9 \mathrm{kHz}$ frequency region and account for $25 \%$ of the total number of collicular neurons sampled (Table 1). Second, there is a disproportionately large number of neurons tuned to frequencies between 83.0 to $84.5 \mathrm{kHz}$. 96 neurons, or $16 \%$ of the total population, had BFs within this $1.5 \mathrm{kHz}$ band (Table 1). The third noteworthy feature is the precipitous decline in the number of neurons tuned to frequencies between 84.6 to $86.0 \mathrm{kHz}$ and the uniformly low number of cells having BFs above $86.0 \mathrm{kHz}$. Only $7 \%$ of the neurons had BFs between 84.6 and $88.0 \mathrm{kHz}$ (Table 1).

The number of neurons with best frequencies within one $\mathrm{kHz}$ bands, expressed as number of neu- rons per octave, is plotted on a scale of best frequencies in Fig. 1 C. This graph shows the unproportionately high density of neurons in the narrow frequency band from 83.0 to $84.5 \mathrm{kHz}$ coinciding with that of the reference frequencies. This density distribution of BFs in the inferior colliculus has a striking resemblance to the innervation density of the basilar membrane (Bruns and Schmieszek, in press).

In Fig. 2 is shown the distribution of thresholds for neurons tuned to the $77-91 \mathrm{kHz}$ band. The important feature of this figure is the absence of any drastic shift in the range of thesholds across frequencies. There is no discernable difference in the ranges of thresholds for neurons tuned between 77 and $82.5 \mathrm{kHz}$ as compared to those tuned between 83.0 and $84.5 \mathrm{kHz}$. The range of thresholds appears to 
Table 1. The distribution of the best frequencies of collicular neurons among frequency bands. The $\mathrm{CF}$ frequency of the emitted sounds and of the returning echoes during echolocation comprise a frequency band between 78 and $88 \mathrm{kHz} .49 .1 \%$ of all neurons encountered in the inferior colliculus had BFs in this frequency range. The total number of neurons sampled is 591

\begin{tabular}{lllllll}
\hline Best frequency & $9-77.9$ & $78-82.9$ & $83-84.5$ & $84.6-87.9$ & $88-97$ & {$[\mathrm{kHz}]$} \\
Number of neurons & 278 & 152 & 96 & 42 & 23 & \\
Neurons per kHz & 4 & 31 & 64 & 13 & 2.5 & {$\left[(\mathrm{kHz})^{-1}\right]$} \\
\% of total sample & 47.1 & 25.7 & 16.3 & 7.1 & 3.9 & {$[\%]$} \\
\hline
\end{tabular}

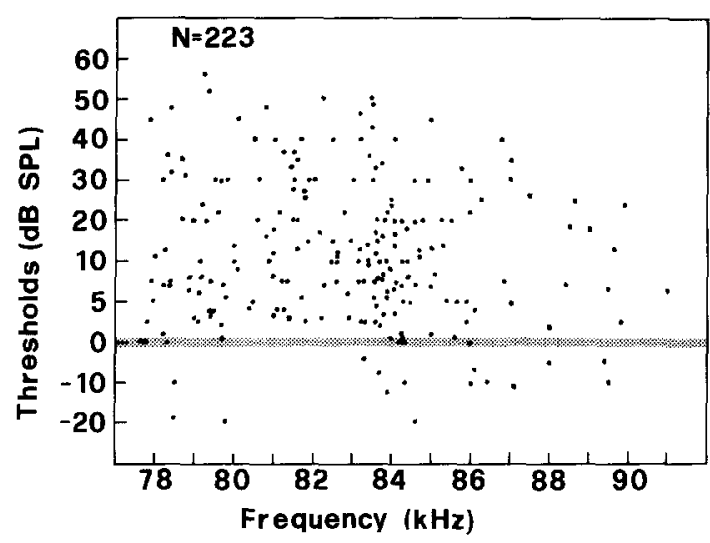

Fig. 2. Intensity threshold at the best frequency for 223 neurons as a function of the best frequencies of the neurons. Only neurons with best frequencies between 77 and $91 \mathrm{kHz}$ are represented. This frequency range covers the range of possible $\mathrm{CF}$-frequencies during echolocation of the greater horseshoe bat

be equally low for neurons having BFs between 84.6 and $91 \mathrm{kHz}$. The majority of neurons, regardless of frequency band considered, have thresholds between 0 and $30 \mathrm{~dB}$ SPL with a much smaller number having higher or lower thresholds.

\section{Discussion}

\section{Relation to Previous Results}

Previous studies have shown that neurons with best frequencies between 78 and $88 \mathrm{kHz}$ constitute about $50 \%$ of the total neuronal population in any auditory center (Möller et al., 1978; Schlegel, 1977; Suga et al., 1976, 1977; Suga and Jen, 1976). However, the frequency bands in which these concentrations of neurons are distributed were only loosely specified. Schlegel (1977) gave the most precise information and reported that $38 \%$ of the neurons were tuned to frequencies $\pm 2.5 \mathrm{kHz}$ around $83 \mathrm{kHz}$ and thus were spread over a $5.0 \mathrm{kHz}$ band of frequencies. However, judging from Fig. 14 of his paper most of the neurons had BFs below the resting frequency. Suga et al. (1976) report data from the cochlear nucleus where, judging from Fig. 4 of their paper, very few neurons were tuned to frequencies below the resting frequency (e.g. $78-83 \mathrm{kHz}$ ) but rather most were concentrated in a $3 \mathrm{kHz}$ band above the resting frequency, between about $83-86$ or $87 \mathrm{kHz}$.

In none of these studies were the brains histologically examined nor was there any mention of attempts to systematically sample the structure under study. This is an important consideration because the tonotopic organization of auditory regions in long CF-FM bats departs markedly from the organization seen in other types of bats and other mammalian orders (Suga and Jen, 1976; Pollak and Schuller, 1978). For example, the inferior colliculus of the horseshoe bat has two physiologically distinct regions, one dorsal and one ventral. In the dorsal region there is an orderly progression of BFs from about $9 \mathrm{kHz}$ to $77 \mathrm{kHz}$ in the familiar dorsoventral axis (Pollak and Schuller, 1978). The frequencies between $78-88 \mathrm{kHz}$ are represented in the ventral portion and are organized along an anterio-posterior axis such that there is a systematic progression of BFs from about $78 \mathrm{kHz}$, in the anterior region, to about $86-88 \mathrm{kHz}$ in the most posterior region. That is, within a particular dorsoventral axis, the full range of neurons with best frequencies below $78 \mathrm{kHz}$ would be more or less represented in the dorsal region but all neurons with BFs above $78 \mathrm{kHz}$ encountered in the more ventral portion would have nearly the same BFs. As a consequence, failure to adequately sample, say the more anterior region of the IC, would result in a sample having a paucity of neurons tuned between $78-80 \mathrm{kHz}$ but in which a disproportionately large number of neurons tuned to $82-85 \mathrm{kHz}$ would be obtained. Assuming the tonotopic arrangement of the cochlear nucleus also differs from the conventional organization, a similar argument could be advanced for the study of this nucleus. Apparently then, the net result of the data from the studies cited above is to represent a fairly accurate picture of the proportional representation of the $78-88 \mathrm{kHz}$ frequencies but they also create a confused view of exactly how the $78-88 \mathrm{kHz}$ neurons are distributed above and below the bat's resting frequency. 
An unsystematic sample may possibly have had a more serious effect upon the distribution of thresholds for the various frequencies. In the study of the horseshoe bat's cochlear nucleus, for example, low thresholds were found for units tuned between $83-91 \mathrm{kHz}$, a finding similar to our own, whereas the neurons tuned between $75-82 \mathrm{kHz}$ had considerably higher thresholds (see Fig. 3, Suga et al., 1976).

However, very few neurons were obtained that were tuned to frequencies of $75-82 \mathrm{kHz}$ and it might well have been that the neurons which were sampled had thresholds between 20-50 dB SPL, thresholds quite commonly encountered for all frequencies. Had a larger sample of neurons tuned to $75-82 \mathrm{kHz}$ been obtained a quite different picture of the threshold distribution might have emerged.

\section{Functional Implications}

A question that has long puzzled us concerns the functional advantage of Doppler-shift compensation. One hypothesis holds that, since neurons tuned to the reference frequency have lower thresholds than do neurons tuned to higher neighboring frequencies, the stabilization of the echo at a frequency to which the bat is most sensitive confers a sensitivity advantage. The results of the present study, as well as that of Suga et al. (1976), would seem to render this hypothesis untenable since many neurons tuned to the frequencies above the reference frequency have thresholds ranging from $-5 \mathrm{~dB}$ to $+10 \mathrm{~dB}$ SPL. But even without these data, the sensitivity hypothesis suffers from internal inconsistency. The first perceptible echo heard by a hunting bat is both Doppler-shifted upward and is quite faint. If the bat had poor sensitivity for frequencies above the reference frequency how would the echo be perceived and compensated for?

If enhanced sensitivity is unlikely, what then is the advantage of DSC? The bats in this study had resting frequencies that ranged from $83.5-83.8 \mathrm{kHz}$. When Doppler compensating, these bats would, on the average, hold the echo frequency at about $83.8-84.1 \mathrm{kHz}$, a region to which a disporportionately large number of neuronal elements are tuned. It is with regard to this statement that the quantitative refinement of proportional representation becomes highly significant. The flight speed of horseshoe bats dictates a maximum Doppler-shift of $4-5 \mathrm{kHz}$. If a disproportionately large neuronal population were equally represented within a $3-5 \mathrm{kHz}$ band above the resting frequency there would be no advantage to DSC. An echo, whether Doppler-shifted by $1 \mathrm{kHz}$ or by $4 \mathrm{kHz}$, would be processed by an equal number of specialized neurons. Furthermore, the size of the population responding to the echo would remain constant even after the echo had been compensated for.

A very different result obtains when the number of neurons tuned to a narrow band of frequencies around the reference frequency is substantially increased at the expense of the numbers tuned to neighboring frequencies. Under these conditions, manipulation of the frequencies of the emitted cries confines the echoes to a very narrow frequency band and this behaviour insures the bat that echoes are processed by an exceptionally large number of sharply tuned neurons.

\section{What Information is Conveyed by and Extracted from the Echo CF Component?}

As mentioned in the introduction, there are two major hypotheses concerning the nature of the information derived from the $\mathrm{CF}$ component: 1) relative velocity information; and 2) prey recognition. It should be stressed that these are not considered to be mutually exclusive. Furthermore, judging from published reports, many investigators consider it to be unequivocal that long CF-FM bats measure relative velocity from Doppler-shift information. We believe the relative velocity hypothesis to be incorrect.

There are two questions relating to velocity measurement which have apparently become fused. The first question is whether DSC is necessary for evaluations of frequency differences while the second is whether the bat is really measuring the relative velocity between itself and its target with evaluations of Doppler-shift information.

\section{A. Is DSC Necessary for Frequency Evaluations?}

The fact that long CF-FM bats Doppler-compensate demonstrates that these animals are evaluating frequency differences. But exactly which frequency difference the bat is primarily concerned with is a question that has not been answered but is of critical importance. There are three frequencies to be considered : 1) the frequency of the emitted CF component; 2) the frequency of the echo CF component; and 3) the bat's reference frequency. There seems to be little reason to compare the difference between the pulse and the reference frequencies so we shall not consider this comparison further. That leaves two possibilities: 1) If the bat is evaluating the difference between its reference frequency and the echo frequency in order to determine by how much it must lower its pulse frequency to cause stabilization of 
the echo at the reference frequency, then the value of DSC becomes apparent; 2) However, if the bat is evaluating the difference between the pulse and the echo frequencies the advantage of relationship of DSC for this analysis becomes obscure.

Let us assume for the moment that the long $\mathrm{CF}$ FM bats are primarily concerned with measuring the frequency difference between the pulse and echo $\mathrm{CF}$ components. A bat hunting an insect will, at some point in the echolocation cycle, receive an echo whose frequency is Doppler-shifted well above the bat's reference frequency. Perception of this Doppler-shifted echo will result in the bat lowering the frequency of its subsequent pulses in order to equate the echo frequency with its own reference frequency. Prior to compensation, the population of neurons responding to an echo Doppler-shifted by $2-4 \mathrm{kHz}$ above the resting frequency is substantially smaller than the population tuned to the resting frequency (see Fig. 1). On the other hand, once compensation has been achieved, and the echo frequency is equal to the reference frequency, the number of neurons devoted to the pulse becomes markedly smaller than the number devoted to the echo. In short, there will be always a significant mismatch in the size of populations devoted to processing the pulse as compared to the echo. Thus, it would appear that the precision of frequency analysis is much more related to the tuning sharpness of at least an adequately represented responding population than it is to the absolute number of responding elements. If this argument is correct, then frequency analysis should be about as accurate before as after compensation and the advantage of DSC for frequency analysis becomes difficult to understand.

\section{B. Are Long CF-FM Bats Evaluating Velocity Via Doppler Effects?}

The second question is fundamental to interpretations of information processing in long CF-FM bats and asks whether the bat is actually measuring the relative velocity between itself and its target by evaluating the amount of Doppler shift in the echo CF component. This is a question which is very difficult to prove any way or another. However, we suggest that just because the animal is highly sensitive to Doppler shifts does not necessarily mean that the bat is using Doppler information for evaluations of its relative flight speed.

There are two considerations which make us question the velocity hypothesis. The first is that long CF-FM bats obtain accurate range information from the analysis of the time delay between the FM com- ponents of the pulse and the echo, just as FM bats do (Simmons, 1973). Therefore, relative velocity can be, and probably is, obtained from estimates of range closing, exactly as FM bats must obtain relative velocity information. This, of course, does not preclude the obtainment of relative velocity from the $\mathrm{CF}$ component but it does argue that there is another source of precise velocity estimates.

Since relative velocity is conveyed by different parameters of the two components of the biosonar signal, we wonder which component is used for measuring relative velocity or does the bat use both sources of information? It is interesting, in this context, to note that the bats used in the DSC experiments were receiving conflicting velocity information from the $\mathrm{CF}$ and from the FM components. In the DSC experiments (Schuller et al., 1974; Simmons, 1974), for example, the played back echoes contained both the CF and FM components but the time delay between the pulse and echo was held constant and thus did not introduce changes in the apparent distance to the 'target'. When Doppler shifts were imposed on the CF component the bat fully compensated for the Doppler shifts. In this case, then, distance information in the FM component "informed" the bat that the target was stationary whereas the CF component "informed" the bat that the target was moving with a velocity of e.g. $4 \mathrm{~m} / \mathrm{s}$.

The very essence of the velocity hypothesis requires a certain interpretation of these data - namely, that the bat simply ignored the velocity information conveyed by changes (in this case, the lack of changes) in distance evaluations and focused only on the Doppler shifts of the CF component. But another interpretation is equally valid - namely, that the bat simply stabilized the echoes at its reference frequency but was not assessing relative velocity. The relationship between relative velocity and frequency changes, in this view, is coincidential. The important point is that there is no way of distinguishing between these interpretations because the experiments were not designed to tease out which component the bat uses for velocity estimates. The assertion that DSC demonstrates Doppler-sensitivity is fact; the assertion that DSC demonstrates velocity measurement is inference and is widely accepted only because of its appeal and not because the supporting evidence is compelling or even strong.

The second consideration which causes us to question the relative velocity hypothesis is much more serious and concerns the assumption that the narrow bandwidth of the CF component renders the signal well suited for velocity measurements. To be sure, horseshoe bats can Doppler-compensate to an accuracy of $\pm 50-100 \mathrm{~Hz}$ when the echoes are of narrow 
bandwidth but it has not been shown that the same precision can be attained while the bat is hunting insects. With regard to echoes, it should be pointed out that the echoes from a flying insect are considerably broader in bandwidth than are tone bursts. Sounds reflected from insects contain periodic frequency and amplitude modulations created as the long CF component strikes and is reflected from the moving wings. The beating wings of a small moth for example, can easily cause frequency modulations to $\pm 500 \mathrm{~Hz}$ around the carrier frequency (Henson and Goldman, 1976). Moreover, insects vary considerably in terms of both the length of their wings and their wing beat frequencies. Both of these features have profound effects on the depth or amount of frequency modulation and hence the bandwidth of the echoes. In this context, wing beats producing frequency modulations of $\pm 500 \mathrm{~Hz}$ introduce an ambiguity of $\pm 1 \mathrm{~m} / \mathrm{s}$ on velocity measurements which is significant considering that horseshoe bats fly at speeds of $4-6 \mathrm{~m} / \mathrm{s}$ (Schnitzler, 1973). Under these conditions, a degree of compensation, and hence an inferred velocity resolution, as accurate as that obtained in laboratory studies with tone bursts is highly unlikely.

\section{The Prey Recognition Hypothesis}

A number of investigators have suggested that the modulation patterns imposed on the CF component of the echo by the beating wings of an insect can convey information about the nature of the target (Schuller, 1972; Schnitzler, 1973, 1978; Henson and Goldman, 1976). Recently some impressive evidence has appeared which strongly supports the prey recognition hypothesis. Goldman and Henson (1977) have shown that the long CF-FM bat, Pteronotus $p$. parnellii, readily pursues and captures free flying and tethered insects or mechanical models when the wings are rapidly moving but they are not attracted to insects or mechanical models when the wing beats are slow. Furthermore, the mustache bat is selective in choice of which insects, among a population having fast wing beats, it will track and capture. Several species of beetles and moths are ignored whereas other species of moths and beetles are vigorously pursued and eaten.

Complementing the behavioural studies are neurophysiological data which demonstrate that neurons in the periphery (Suga et al., 1975) as well as in the inferior colliculus (Schuller, 1972; Pollak and Schuller, 1978; Schuller, 1979) and cortex (Schnitzler, 1978) of long CF-FM bats encode amplitude and frequency modulation patterns with great precision.

\section{$V$. Advantages of Doppler-Shift Compensation for Target Characterization}

If the behavioral and neurophysiological results given above are combined with the data from the present study, we are led to the following hypothesis concerning the functional advantage of DSC for information processing. Under natural conditions the bat seeks to characterize its target by extracting the information contained in the modulation patterns of the echo $\mathrm{CF}$ component. By Doppler compensating the bat effectively stabilizes the echo spectrum within a frequency region to which a disproportionately large number of neural elements are tuned, each well suited to encode some component of the modulated signal. To accomplish this, the bat needs only match the spectrum of the echo to the $1.5 \mathrm{kHz}$ band around its reference frequency. Whether in so doing the bat obtains a rough estimate of relative velocity is something that cannot presently be ruled out. However, we wish to stress that we, along with Schnitzler (1973), believe the important relation to be between the echo and reference frequencies, from which velocity information cannot be obtained, rather than a precise comparison of emitted and echo frequencies, which is required for an analysis of relative velocity. Stated differently, a compensation accuracy of $\pm 50-100 \mathrm{~Hz}$ is apparently an advantage for matching the relatively broad band modulated signal close to the reference frequency but does not cancel the inaccuracy introduced by the modulation in the echoes for a velocity measurement. The bat needs only perceive a significant discrepancy between the higher echo frequencies and the reference frequency and adjust its pulses such that a large portion of the echo spectrum falls within the $83.0-84.5 \mathrm{kHz}$ region in order for these neurons to be used effectively.

Here we regard Doppler-shift compensation not as a mechanism for enhancing sensitivity nor as a mechanism for extracting relative velocity information but rather as a mechanism for "tracking" echoes.

Viewed in this way, the acoustic system of Rhinolophus bears a marked resemblance to the visual system: eye movements (i.e. Doppler-shift compensation) keep images of interest (i.e. echoes) focused on the fovea (i.e. the 83.0 to $84.5 \mathrm{kHz}$ region) and as a consequence the ability to both detect and to resolve image (i.e. target) features are enhanced. This functional acoustic fovea from 83.0 to $84.5 \mathrm{kHz}$ corresponds to a morphological fovea found in the basal part of the basilar membrane in Rhinolophus. There the frequency range from 83 to $86 \mathrm{kHz}$ is represented in a vastly expanded fashion covering the same length of the basilar membrane commonly used for a complete octave (e.g. 40 to $80 \mathrm{kHz}$; Bruns, 1976). 
Thus in the acoustic as in the visual system a fovea is realized on the level of the receptors. Moreover, as in the visual system an unproportionately high percentage $(21 \%$, Bruns and Schmieszek, in press) of afferent fibres innervate the foveal region and as in the visual system the foveal overrepresentation is maintained through all central nervous nuclei up to the cortex.

The encouragement and suggestions of Gerhard Neuweiler (University Frankfurt) and the suggestions of Bill Giesler (University of Texas) are gratefully acknowledged.

\section{References}

Bruns, V.: Peripheral auditory tuning for fine frequency analysis by the CF-FM bat, Rhinolophus ferrumequinum. I. Mechanical specialization of the cochlea. J. Comp. Physiol. 106, 77-86 (1976a)

Bruns, V.: Peripheral auditory tuning for fine frequency analysis by the CF-FM bat, Rhinolophus ferrumequinum. II. Frequency mapping in the cochlea. J. Comp. Physiol. 106, $87-97$ (1976b)

Bruns, V., Schmieszek, E.: Regional differentiations of the innervation pattern of the cochlea of the Greater Horseshoe bat. (in press) (1979)

Goldman, L.J., Henson, O.W.: Prey recognition and selection by the constant frequency bat, Pteronotus p. parnellit. Behav. Ecol. Sociobiol. 2, 411-419 (1977)

Grinnell, A.D. : Neural processing mechanisms in echolocating bats, correlated with differences in emitted sounds. J. Acoust. Soc. Am. 54, 147-155 (1973)

Harnischfeger, G. : An improved method for extracellular marking of electrode tip positions in nervous tissue. J. Neurosci. Meth., (in press) (1979)

Henson, O.W., Jr., Goldman, L.J.: Prey detection and physiological aspects of the cochlea in the bat, Pteronotus $p$. parnellii. Anat. Rec. 184, 425 (1976)

Long, J.R., Schnitzler, H.-U.: Behavioural audiograms from the bat, Rhinolophus ferrumequinum. J. Comp. Physiol. 100, 211-219 (1975)

Möller, J., Neuweiler, G., Zöller, H.: Response characteristics of inferior colliculus neurons of the awake CF-FM bat, Rhinolophus ferrumequinum. I. Single-tone stimulation. J. Comp. Physiol. 125, 217-225 (1978)

Neuweiler, G.: Neurophysiologische Untersuchungen zum Echoortungssystem der Großen Hufeisennase Rhinolophus ferrumequinum Schreber, 1774. Z. Vergl. Physiol. 67, 237-306 (1970)

Neuweiler, G., Vater, M.: Response pattern to pure tones of cochlear nucleus units in the CF-FM bat, Rhinolophus ferrumequinum. J. Comp. Physiol. 115, 119-133 (1977)

Pollak, G., Henson, O.W., Jr., Novick, A.: Cochlear microphonic audiograms in the "pure tone" bat Chilonycteris parnellii parnellii. Science 176, 66-68 (1972)

Pollak, G., Schuller, G.: Tonotopic organization and response patterns to frequency modulated signals in the inferior colliculus of Horseshoe bats. Soc. Neurosci. Abstr. 4, 9 (1978)

Schlegel, P.: Directional coding by binaural brainstem units of the CF-FM bat, Rhinolophus ferrumequinum. J. Comp. Physiol. 118, 327-352 (1977)

Schnitzler, H.-U.: Discrimination of thin wires by flying horseshoe bats (Rhinolophidae). In: Animal sonar systems, Vol. I, Busnel, R.G. (ed.), pp. 69-87. Jouy-en Josas: Laboratoire de Physiologie Acoustique 1967

Schnitzler, H.-U.: Die Ultraschall-Ortungslaute der HufeisenFledermäuse (Chiroptera, Rhinolophidae) in verschiedenen Orientierungssituationen. Z. Vergl. Physiol. 57, 376-408 (1968)

Schnitzler, H.-U.: Echoortung bei der Fledermaus Chilonycteris rubiginosa. Z. Vergl. Physiol. 68, 25-38 (1970)

Schnitzler, H.-U.: Control of Doppler-shift compensation in the Greater Horseshoe bat, Rhinolophus ferrumequinum. J. Comp. Physiol. 82, 79-92 (1973)

Schnitzler, H.-U.: Die Detektion von Bewegungen durch Echoortung bei Fledermäusen. Verh. Dtsch. Zool. Ges. 1978, 16-33 (1978)

Schuller, G.: Echoortung bei Rhinolophus ferrumequinum mit frequenzmodulierten Lauten. Evoked potentials im Colliculus inferior. J. Comp. Physiol. 77, 306-331 (1972)

Schuller, G.: Coding of small sinusoidal frequency and amplitude modulations in the inferior colliculus of 'CF-FM' bat, Rhinolophus ferrumequinum. Exp. Brain Res. 34, 117-132 (1979)

Schuller, G., Beuter, K., Schnitzler, H.-U.: Response to frequency shifted artificial echoes in the bat Rhinolophus ferrumequinum. J. Comp. Physiol. 89, 275-286 (1974)

Simmons, J.A.: The resolution of target range by echolocating bats. J. Acoust. Soc. Am. 54, 157-173 (1973)

Simmons, J.A.: Response of the Doppler echolocation system in the bat, Rhinolophus ferrumequinum. J. Acoust. Soc. Am. 56, 672-682 (1974)

Simmons, J.A., Howell, D.J., Suga, N.: Information content of bat sonar echoes. Am. Sci. 63, 204-215 (1975)

Simmons, J.A., Fenton, M.B., O'Farrell, M.J.: Echolocation and Pursuit of Prey by bats. Science 203, 16-21 (1979)

Suga, N.: Specialization of the auditory system for reception and processing of species-specific sounds. Fed. Proc. 37, 2342-2354 (1978)

Suga, N., Jen, Ph., H.-S.: Disproportionate tonotopic representation for processing CF-FM sonar signals in the mustache bat auditory cortex. Science 194, 542-544 (1976)

Suga, N., Jen, Ph., H.-S.: Further studies on the peripheral auditory system of ' $\mathrm{CF}-\mathrm{FM}$ ' bats specialized for fine frequency analysis of Doppler-shifted echoes. J. Exp. Biol. 69, 207-232 (1977)

Suga, N., O'Neill, W.E.: Mechanisms of echolocation in bats: comments on the neuroethology of the biosonar system of ' $\mathrm{CF}$ FM' bats. Trends Neurosci. 1, 35-38 (1978)

Suga, N., Simmons, J.A., Jen, Ph., H.-S. : Peripheral specialization for fine frequency analysis of Doppler-shifted echoes in the auditory system of the CF-FM bat Pteronotus parnellii. J. Exp. Biol. 63, 161-192 (1975)

Suga, N., Neuweiler, G., Möller, J.: Peripheral auditory tuning for fine frequency analysis of the CF-FM bat, Rhinolophus ferrumequinum. IV. Properties of peripheral auditory neurons. J. Comp. Physiol. 106, 111-125 (1976) 